\title{
Lithium ist Quetiapin überlegen
}

Fragestellung: Welche Effekte hat eine Erhaltungstherapie mit Lithium oder Quetiapin auf die Stabilisierung von depressiven und manischen Symptomen, das allgemeinen Funktionsniveau und die Lebensqualität bei Patienten mit einer ersten Episode einer Manie?

Hintergrund: Lithium und Quetiapin sind Standardmedikationen zur Erhaltungstherapie bei bipolarer Störung. Bisher gibt es allerdings nur wenige Studien, die die Effekte beider Substanzen in der Erhaltungstherapie direkt miteinander verglichen haben.

Patienten und Methodik: Die Autoren randomisierten 61 Patienten mit einer akuten ersten Episode einer Manie mit psychotischen Symptomen, die unter einer Kombinationstherapie von Quetiapin und Lithium remittierten, auf zwei Behandlungsarme: 31 Patienten wurden auf eine Erhaltungstherapie mit Quetiapin und 30 auf eine Erhaltungstherapie mit Lithium

Berk M, Daglas R, Dandash 0 et al. Quetiapine vs. lithium in the maintenance phase following a first episode opf mania: randomized controlled trial. Brit J Psychiatry 2017; 210: $413-21$ randomisiert. Nach zwölf Monaten verblieben noch 26

Lebensqualität wurden mit üblichen Skalen gemessen. Der mittlere Lithium-Spiegel betrug 0,6 mmol/l, die mittlere QuetiapinDosis 437,5 mg, wobei eine Maximaldosis von $800 \mathrm{mg} / \mathrm{Tag}$ möglich war. Haupt-Outcomes waren Verlauf der depressiven oder manischen Symptomatik, der psychotischen Symptomatik, des allgemeinen Funktionsniveaus und der Lebensqualität innerhalb eines Jahres.

Ergebnisse: In allen Analysen zeigte sich Lithium Quetiapin überlegen. Bezüglich manischer Symptome zeigten sich im Verlauf keine Unterschiede, nach neun Monaten waren allerdings die manischen Symptome unter Quetiapin-Behandlung stärker ausgeprägt als unter Lithium.

Bezüglich der depressiven und psychotischen Symptomatik sowie dem allgemeinen Funktionsniveau zeigte sich im Verlauf unter Quetiapin eine signifikante Verschlechterung, die unter Lithium-Behandlung nicht beobachtet wurde. Nach zwölf Monaten zeigte sich unter der Quetiapin-Behandlung eine stärkere Ausprägung von depressiven und psychotischen Symptomen und ein schlechteres Funktionsniveau als unter der Behandlung mit Lithium. und 24 in der LithiumBehandlungsgruppe. Die depressive und manische Symptomatik sowie das allgemeine Funktionsniveau und die
Schlussfolgerungen: Patienten mit psychotischer Manie, die erfolgreich mit Lithium und Quetiapin behandelt wurden, profitieren eher von einer Erhaltungstherapie mit Lithium als von einer mit Quetiapin.

\section{- Kommentar von Klaus Lieb, Mainz}

\section{Lithium unverändert erste Wahl bei Erhaltungstherapie}

Es handelt sich hier um eine kleine Studie, deren Ergebnisse grundsätzlich vorsichtig zu interpretieren sind. In zwei anderen Studien konnten keine Unterschiede zwischen Lithium und Quetiapin bezüglich der Langzeitstabilisierung gefunden werden, wobei diese unterschiedliche Designs aufwiesen.

In der CHOICE-Studie [1] wurde eine Add-on-Therapie mit Quetiapin oder Lithium durchgeführt, wobei sich keine Unterschiede im Outcome zeigten.

In der Studie von Weisler et al. [2] wurden mehr als 1.000 Patienten mit manischen, depressiven oder gemischten Episoden auf Quetiapin stabilisiert und dann auf eine Erhaltungstherapie mit Quetiapin, Lithium oder Placebo randomisiert. Hier zeigten sich keine Unterschiede in der Verhinderung von neu aufgetretenen Episoden.

Da insbesondere die letzte Studie deutlich mehr Patienten eingeschlossen hat, spricht doch einiges dafür, dass man die Unterschiede zwischen Quetiapin und Lithium vorsichtig interpretieren muss. Allerdings traten die Unterschiede in der hier vorgelegten Studie erst nach neun und zwölf Monaten auf, während zum Beispiel die CHOICE-Studie nur eine Beobachtungszeit von sechs Monaten hatte. Bemerkenswert an der hier vorgelegten Studie ist, dass offenbar auch psycho- tische Symptome unter Lithium besser stabilisiert wurden als unter Quetiapin.

Welche klinische Konsequenz hat nun diese Studie? Sie zeigt erneut das breite Spektrum der stabilisierenden Wirkung von Lithium, sodass aus meiner Sicht Lithium unverändert die erste Wahl zur Erhaltungs- und Rezidivprophylaxe bei manischen und depressiven Episoden bleiben sollte.


Prof. Dr. med. Klaus Lieb, Mainz

Direktor der Klinik für Psychiatrie und Psychotherapie, Universitätsmedizin Mainz E-Mail: klaus.lieb@unimedizin-mainz.de 